\title{
$11 \beta$-Hydroxysteroid dehydrogenase type 2 in zebrafish brain: a functional role in hypothalamus-pituitary-interrenal axis regulation
}

\author{
Sarah L Alderman and Mathilakath M Vijayan \\ Department of Biology, University of Waterloo, Waterloo, Ontario, Canada \\ (Correspondence should be addressed to M M Vijayan who is now at Department of Biological Sciences, University of Calgary, 2500 University Drive N.W., \\ Calgary, Alberta, Canada T2N 1N4; Email: matt.vijayan@ucalgary.ca)
}

\begin{abstract}
The type 2, 11ß-hydroxysteroid dehydrogenase (Hsd11b2) converts active glucocorticoids to their inactive derivatives (e.g. cortisol to cortisone). In most vertebrates, Hsd11b2 is essential for conferring aldosterone-specific actions in mineralocorticoid target tissues and for protecting glucocorticoid-sensitive tissues during stress. However, teleosts do not synthesize aldosterone, and the function of $\mathrm{Hsd} 11 \mathrm{~b} 2$ is poorly defined. The distribution of $\mathrm{Hsd} 11 \mathrm{~b} 2$ in nonmammalian brain is also largely unexplored. We tested the hypothesis that modulation of brain Hsd11b2 activity is involved in stressor-mediated cortisol regulation in zebrafish (Danio rerio). In adult zebrafish, the stress effect on Hsd11b2 expression in the brain was tested using acute air exposure followed by recovery over a 24-h period. hsd11b2 transcripts were found in nearly all peripheral tissues
\end{abstract}

examined, and a spatial map of its mRNA abundance in unstressed zebrafish brain revealed extensive distribution. Stressor exposure increased the conversion of ${ }^{3} \mathrm{H}$-cortisol to ${ }^{3} \mathrm{H}$-cortisone indicating enhanced $\mathrm{Hsd} 11 \mathrm{~b} 2$ activity in zebrafish brain. Promoter analysis of zebrafish hsd $11 b 2$ gene revealed putative sites for cortisol-mediated transcriptional regulation of this gene. Furthermore, inhibition of Hsd11b2 activity by $18 \beta$-glycyrrhetinic acid resulted in elevated whole-body cortisol levels and preoptic area mRNA abundance of corticotropin-releasing factor and mineralocorticoid receptor. Taken together, our results underscore an important role for brain Hsd11b2 involvement in the negative feedback regulation of cortisol poststress in zebrafish.

Journal of Endocrinology (2012) 215, 393-402

\section{Introduction}

Cortisol is the primary glucocorticoid in teleosts and is released from interrenal cells (adrenal gland homolog) during stress following activation of the hypothalamus-pituitaryinterrenal (HPI) axis. During stress, afferent inputs to the hypothalamus stimulate the release of corticotropin-releasing factor $(\mathrm{CRF})$ from the preoptic area (POA), which in turn stimulates the pituitary to secrete ACTH. Circulating ACTH then signals the interrenal cells to synthesize and secrete cortisol (Wendelaar Bonga 1997, Mommsen et al. 1999). The primary function of cortisol is to increase glucose bioavailability in order to meet the increased energy demands of stress; however, it can impact the functions of most body systems (Wendelaar Bonga 1997, Mommsen et al. 1999).

Negative feedback regulation of HPI axis activity is mediated by cortisol at all levels of the axis, including suppression of CRF-mediated ACTH release (Fryer \& Peter 1977); however, the mechanisms governing cortisol feedback in the brain remain unclear in teleosts. In mammals, diurnal hypothalamus pituitary adrenal (HPA) axis activity is regulated by glucocorticoid-negative feedback acting through the highaffinity mineralocorticoid receptor (MR), whereas stressor levels of glucocorticoids exert negative feedback by binding to the lower affinity glucocorticoid receptor (GR; Yao \& Denver 2007). In teleosts, we recently showed that GR is involved in the cortisol response to stress (Alderman et al. 2012), but the mechanism involved in the negative feedback regulation of cortisol is not known. Furthermore, the potential contribution of central enzymatic inactivation of cortisol in the regulation of HPI axis function has not been investigated.

At the target tissue level, a key player that regulates cortisol bioavailability is the enzyme $11 \beta$-hydroxysteroid dehydrogenase (Hsd11b) that catalyzes the conversion of this steroid to its active or inactive forms (Krozowski 1999). While the type 1 isozyme (Hsd11b1) increases local cortisol concentrations by converting inert cortisone to cortisol, the type 2 form (Hsd11b2) works in the reverse direction to inactivate cortisol (Krozowski 1999). In mammals, Hsd11b2 activity is important in glucocorticoid-sensitive tissues, such as the fetal brain (Wyrwoll et al. 2011). Hsd11b2 is also critical in preventing inappropriate activation of $\mathrm{MR}$, which binds both glucocorticoids and aldosterone with similar or greater affinity (Krozowski 1999). Of note, the capacity to synthesize aldosterone does not appear until after the divergence of lobe-finned fishes from ray-finned fish (Colombo et al. 2006), 
and a specific ligand for MR, other than cortisol, with a physiological role has yet to be confirmed in teleosts; however, 11-deoxycorticosterone is a candidate for consideration (Sturm et al. 2005, Stolte et al. 2008).

Studies have shown that $h s d 11 b 2$ transcripts are broadly expressed in peripheral tissues of teleost fish (Jiang et al. 2003, Kusakabe et al. 2003, Rasheeda et al. 2010), but its physiological role is far from clear. A local role in testicular development and gametogenesis related to 11-ketotestosterone production is the only described function to date (Kusakabe et al. 2003, Rasheeda et al. 2010). Furthermore, the presence and function of Hsd11b2 in the teleost CNS has not been investigated. The purpose of this study was to investigate the capacity of the teleost brain to regulate cortisol bioavailability by characterizing the expression pattern and activity of Hsd11b2 in response to stress. Specifically, we tested the hypothesis that Hsd11b2 plays a critical role in cortisol regulation in response to stress. To this end, adult zebrafish were subjected to an acute air exposure either with or without the Hsd11b2 inhibitor, 18ß-glycyrrhetinic acid (Vicker et al. 2004). We quantified changes in whole-body cortisol content, and Hsd11b2 activity and mRNA abundances of $h s d 11 b 2, c r f, g r$, and $m r$ in zebrafish brain. The results show, for the first time, a key role for central Hsd11b2 in regulating HPI axis function in adult fish.

\section{Materials and Methods}

\section{Animals}

Adult wild-type zebrafish (Danio rerio) were obtained from a local supplier and maintained at $28{ }^{\circ} \mathrm{C}$ and $14 \mathrm{~h} \mathrm{light:} 10 \mathrm{~h}$ darkness with twice daily feedings of commercial pellets. Care and use of the animals were approved by the University of Waterloo Animal Care Committee as per the principles of the Canadian Council for Animal Care.

\section{Tissue distribution}

RT-PCR To assess the tissue distribution of hsd11b2, one adult male zebrafish was terminally anesthetized in $2 \mathrm{ml} / 1$ 2-phenoxyethanol and the brain, eye, gill, heart, liver, head kidney, posterior kidney, muscle, swim bladder, and gut were excised and snap-frozen. Total RNA was extracted using TRIzol (Life Technologies Inc., Burlington, ON, Canada) and treated with DNase 1 (Fermentas Biotechnology Tools, Burlington, ON, Canada) as per manufacturers' instructions. cDNA was synthesized from 500 ng RNA with the High-Capacity cDNA Synthesis Kit (Life Technologies) as per manufacturer's instructions. RT-PCR reactions contained $1 \times$ buffer, $2 \mathrm{mM} \mathrm{MgSO} 4,200 \mu \mathrm{m}$ dNTPs, and 1 unit Taq polymerase (Bio Basic Canada, Inc., Markham, ON, Canada), plus $200 \mathrm{nM}$ each gene-specific primer (hsd11b2: F, 5'-CTGAGTTGGAACGTGTGAGA-3'; R, 5'-AGCACAGTCGCAAACACTTC- $3^{\prime}$; for $\beta$-actin see Alsop \&
Vijayan (2008)) and $1 \mu \mathrm{l}$ cDNA or water (negative control) in a total volume of $25 \mu \mathrm{l}$. Standard thermal cycling parameters were followed for 30 ( $\beta$-actin) or 40 cycles (hsd11b2) at primer-specific annealing temperatures and amplicon-specific extension times. Products were viewed by gel electrophoresis.

In situ hybridization Following Thisse \& Thisse (2008), PCR was used to generate $580 \mathrm{bp}$ DNA fragments of the hsd11b2 gene (GenBank accession no.: NM 212720.1) with T7 recognition sites at either the $5^{\prime}-$ (F, 5'-TAATACGACTCACCCGAGCAGGCACA-3'; R, as in RT-PCR) or the $3^{\prime}$-end ( $\mathrm{F}$, as in RT-PCR; $\mathrm{R}, 5^{\prime}$-TAATACGACTCACTCCTTCCAGGTTG- $3^{\prime}$ ). The amplified region of the gene, which contained $230 \mathrm{bp}$ of the $5^{\prime}$-UTR and was not homologous to other zebrafish transcripts (Blast analysis), was confirmed by sequencing and then used as template to synthesize DIG-labeled cRNA probes with T7 polymerase. Probes were diluted to $0.75 \mu \mathrm{g} / \mathrm{ml}$ in hybridization buffer (Thisse \& Thisse 2008) and stored in slide mailers at $-20{ }^{\circ} \mathrm{C}$ until use. Nine adult zebrafish of mixed sex were terminally anesthetized as described earlier. The brains were exposed dorsally and the head was fixed overnight at $4{ }^{\circ} \mathrm{C}$ in $4 \%$ paraformaldehyde in PBS ( $\mathrm{pH} 7 \cdot 4)$. The fixed brains were carefully dissected and processed for cryosectioning as described previously (Alderman \& Bernier 2007). Hybridization and detection of sense and antisense cRNA probes was performed exactly as described previously (Alderman \& Bernier 2007) on serial $12 \mu \mathrm{m}$ cryosections. Brightfield photomicrographs of all sections were acquired from a Nikon AZ100 using NIS-Elements BR3.10. Adobe Photoshop was used to match selected photomicrographs for brightness and contrast in order to improve the quality of presentation; no adjustments to positive staining were made. Corel Draw was used to generate line drawings from original photomicrographs.

\section{Stressor exposure}

Experiment 1 To establish the in vivo regulation of brain Hsd11b2 expression and activity in response to stress, adult mixed-sex fish were subjected to a 1-min air exposure and allowed to recover for $20 \mathrm{~min}(n=6), 1 \mathrm{~h}(n=16)$, $4 \mathrm{~h}(n=16)$, and $24 \mathrm{~h}(n=16)$ poststressor exposure as described previously (Fuzzen et al. 2010). At the end of the recovery period, fish were terminally anesthetized as earlier. At 20-min recovery, brains were quickly removed and snapfrozen for subsequent qPCR analysis, and the remaining body was snap-frozen on dry ice for later cortisol analysis. At 1-, 4-, and 24-h recovery, the whole brain was removed and rinsed in ice-cold modified Ringer's $(15 \mathrm{mM} \mathrm{NaHCO}, 2 \mathrm{mg} / \mathrm{ml}$ glucose, $300 \mu \mathrm{g} / \mathrm{ml} \mathrm{BSA}, 15 \mathrm{mM}$ HEPES, $\mathrm{pH} 7 \cdot 4)$, then pooled randomly (four brains/tube, $n=4$ per time point) in $200 \mu \mathrm{l}$ Ringer's for subsequent analysis of Hsd11b2 enzyme activity, and total protein content. The remaining body was snap-frozen on dry ice for later cortisol analysis. A control group of unstressed fish was similarly sampled $(n=6$ for qPCR and cortisol and $n=16$ for Hsd11b2 enzyme activity). 
Experiment 2 In a subsequent experiment, mixed-sex adult zebrafish were divided into 11 aquaria $(n=6 /$ tank) containing either vehicle control $(0.1 \%$ ethanol) or vehicle with $1 \mathrm{mg} / 1$ 18ß-glycyrrhetinic acid (18ß-GA; Sigma-Aldrich Corporation), an Hsd11b inhibitor that preferentially inhibits the type 2 isozyme over the type 1 in vitro in rats (Vicker et al. 2004). The concentration was chosen based on the i.p. LD50 in rodents $(308 \mathrm{mg} / \mathrm{kg}$; Sigma) and also after a preliminary range-finding experiment to determine nonlethal levels in zebrafish. Each aquarium was maintained as a static system at $28^{\circ} \mathrm{C}$ with oxygenation, and a one-third volume water change was performed every $24 \mathrm{~h}$. Fish were housed in treatment tanks for $48 \mathrm{~h}$ and then exposed to air for $1 \mathrm{~min}$ as in Experiment 1. Fish were terminally sampled either before stress ( 0 time) or at 1-, 4-, and 24-h poststressor exposure exactly as described earlier. The preoptic area (POA) of the brain was removed and snap-frozen for subsequent qPCR analysis, and the remaining body was snap-frozen for whole-body cortisol determination. To confirm the efficacy of $18 \beta-G A$ water exposure on Hsd11b2 inhibition, a second group of unstressed fish $(n=6)$ was killed after $48 \mathrm{~h}$ exposure to the drug or solvent. Brains were quickly removed and homogenized in $200 \mu \mathrm{l}$ ice-cold Ringer's (three pooled brains $/ n, n=2$ ) for determination of Hsd11b2 enzyme activity and protein content.

\section{Analyses}

Promoter characterization Putative transcription factor binding sites in the promoter region of zebrafish hsd $11 b 2$ (GenBank accession no.: NC_007118; 2434 bp upstream of transcription start site (TSS)) were identified using three independent softwares: TFsearch (http://www.cbrc.jp/ research/db/TFSEARCH.html), TESS (http://www.cbil. upenn.edu/cgi-bin/tess/tess), and MatInspector (http://www. genomatix.de/en/produkte/genomatix-software-suite.html).

Real-time PCR Total RNA extraction and cDNA synthesis were carried out as described earlier. A subset of samples was also used to generate non-reverse-transcribed (non-RT) controls by omitting the MultiScribe RT enzyme from the cDNA synthesis reaction. Semiquantitative real-time PCR was used to measure changes in whole brain $h s d 11 b 2$ (Experiment 1) and POA $c r f, g r$, and $m r$ (Experiment 2) mRNA abundances following air exposure in duplicate reactions as described previously (Alsop \& Vijayan 2008). Gene-specific primer sequences are as reported in Alsop \& Vijayan (2008). Transcript abundance was calculated by fitting the threshold cycle to the antilog of a standard curve made from serially diluted cDNA, and all samples were normalized to the abundance of elongation factor $1 \alpha$ (ef $1 \alpha$; primer sequences reported in Fuzzen et al. (2010)); the threshold cycle for ef $1 \alpha$ was similar in all samples and hence used for normalization. All non-RT controls failed to amplify confirming the absence of genomic contamination, while single-peaked dissociation curves confirmed specific amplification of a single product.
Hsd11b2 enzyme activity As an initial validation of the protocol and specificity of ${ }^{3} \mathrm{H}$-cortisol conversion to ${ }^{3} \mathrm{H}$-cortisone by $\mathrm{Hsd} 11 \mathrm{~b} 2$, adult zebrafish $(n=8)$ were terminally anesthetized as described earlier. Whole brains were removed and pooled in $400 \mu \mathrm{l}$ ice-cold Ringer's (four brains/tube, $n=2$ ) and homogenized. As a positive control, one ripe ovary from a fish was separately homogenized in $400 \mu \mathrm{l}$ ice-cold Ringer's. Homogenates $(180 \mu \mathrm{l})$ or buffer (blank control) was incubated in the presence of $50 \mathrm{nM}$ ${ }^{3} \mathrm{H}$-cortisol (specificactivity $79 \cdot 28 \mathrm{Ci} / \mathrm{mmol} ; \sim 200000$ c.p.m.) and $1 \mathrm{mM} \mathrm{NAD}{ }^{+}$. Parallel reactions containing $10 \mu \mathrm{M}$ $18 \beta-$ GA were included to confirm the specificity of enzymatic conversion by Hsd11b2. Reactions were incubated for $4 \mathrm{~h}$ at $28^{\circ} \mathrm{C}$ on a shaker, then snap-frozen, and stored at $-20^{\circ} \mathrm{C}$ until steroid extraction. Identical conditions were used to incubate brain homogenates from Experiments 1 and 2.

HPLC Steroids were liquid-liquid extracted three times with $1 \mathrm{ml}$ diethyl ether and the organic phase was evaporated under forced air. Extraction efficiencies averaged 89\%. The dried extracts were reconstituted in $20 \%$ acetonitrile in water and HPLC was performed exactly as described previously (Lowartz et al. 2003, Aluru et al. 2005) using a flow rate of $0.5 \mathrm{ml} / \mathrm{min}$.

To quantify enzymatic conversion of ${ }^{3} \mathrm{H}$-cortisol to ${ }^{3} \mathrm{H}$-cortisone, the contribution of each fraction to the total c.p.m. was calculated. All fractions representing $\geq 2 \%$ total c.p.m. that fell within the range of the retention time for the appropriate standard (cortisol or cortisone) were summed to represent the total amount of ${ }^{3} \mathrm{H}$-cortisol and ${ }^{3} \mathrm{H}$-cortisone in each sample. The percent conversion to ${ }^{3} \mathrm{H}$-cortisone was calculated and adjusted to the protein concentration of the homogenate. Total protein content in the homogenates was measured in duplicate using the bicinchoninic acid protein assay (Pierce, Rockford, IL, USA) according to the manufacturer's instructions, with BSA standards.

Cortisol extraction and quantification Fish from Experiment 1 were thawed on ice, blotted dry, and weighed ( $n=6$ per time point), then homogenized in buffer $\left(80 \mathrm{mM} \mathrm{Na} 2 \mathrm{HPO}_{4}, 20 \mathrm{mM} \mathrm{NaH} \mathrm{PO}_{4}, 100 \mathrm{mM} \mathrm{NaCl}\right.$, and $1 \mathrm{mM}$ EDTA, pH 8.0), and sonicated exactly as described previously (Fuzzen et al. 2010). Briefly, steroids were liquidliquid extracted three times with $1 \mathrm{ml}$ methanol and the organic phase was evaporated overnight in the fume hood. Dried extracts were reconstituted in $3 \mathrm{ml}$ assay buffer (21.4 mM Na $\mathrm{NPO}_{4}, 9 \cdot 3 \mathrm{mM} \mathrm{NaH} \mathrm{PO}_{4}, 0 \cdot 1 \%$ gelatin, and $0 \cdot 01 \%$ thimerosal). Extraction efficiency, determined by spiking homogenates with a known quantity of ${ }^{3} \mathrm{H}$-cortisol, averaged $77 \%$. For Experiment 2, frozen fish bodies were manually ground on dry ice and a weighed quantity of powdered tissue was transferred to a $5 \mathrm{ml}$ glass tube containing $2 \mathrm{ml} / \mathrm{g}$ homogenization buffer. Samples were homogenized, briefly sonicated, and extracted three times with $2 \mathrm{ml}$ diethyl ether. The organic phase was evaporated 
and dried extracts were reconstituted in $500 \mu \mathrm{l}$ assay buffer. Extraction efficiency averaged $81 \%$.

Cortisol content of all extracts was measured in duplicate using a commercially available cortisol ELISA kit (Neogen Corporation, Lexington, KY, USA) according to the manufacturer's instructions exactly as described previously (Gonçalves et al. 2012). The highest reported cross-reactivity of this antiserum with other endogenous steroids is $<16 \%$ (Neogen Corporation). Cortisol values were expressed as ng/g body weight and have been corrected for extraction efficiency.

Statistical analysis All data were subjected to an outlier test before statistical analyses. In total, four data points were $>2 \cdot 0 \times$ interquartile range from the median and were excluded from further analysis. For Experiment 1, a one-way ANOVA was used to determine significant differences in whole-body cortisol levels $(n=5-6)$ and in Hsd11b2 enzymatic activities in brain homogenates $(n=4)$. A Student's unpaired $t$-test was used to determine significant differences in $h s d 11 b 2$ transcript levels in the brain $(n=6)$. For Experiment 2, a two-way ANOVA (time $\times$ treatment) was used to determine differences in whole-body cortisol levels and in crf, gr, and $m r$ transcript abundances in the POA $(n=5-6)$.

\section{Results}

\section{Hsd11b2 distribution}

With the exception of muscle, all adult zebrafish tissues examined amplified hsd11b2 (Fig. 1). Hybridization of antisense $h s d 11 b 2$ riboprobes to transverse brain sections revealed extensive expression throughout the brain from olfactory bulbs to the medulla that was consistent across all nine brains and is summarized in Fig. 2. Serial sections to which sense riboprobes were applied failed to demonstrate hybridization (images not shown), confirming the specificity of antisense labeling. The telencephalon was particularly high in $h s d 11 b 2$ expression along the ventricular regions of both the dorsal and the ventral telencephalic areas (D and V respectively; Fig. 2C). Owing to telencephalic eversion in teleosts, the ventricular region of D is located along the dorsal and lateral surfaces (Wullimann et al. 1996). Positive staining typically formed discrete punctate patterns within defined brain nuclei and zones, including the dorsal nucleus of $\mathrm{V}$
(Fig. 3A), the magnocellular preoptic nucleus (Fig. 3B), posterior tuberal nucleus and ventral periventricular hypothalamus (Fig. 3C), and the tectum opticum (Fig. 3D). Other staining was more diffuse, such as in the olfactory bulbs (Fig. 3E), periventricular gray zone of the optic tectum (Fig. 3D), inferior lobes of the hypothalamus (Fig. 3F), and corpus cerebelli (Fig. 3G). In addition, an intense signal was observed along the peripheral edge of all brain slices (Fig. 3D, $\mathrm{E}$ and F).

\section{Promoter characterization}

Many putative transcription factor binding sites were identified in the 2434 bp upstream of the TSS of hsd11b2. A selection of these sites that were identified by at least two of the three softwares used in the analysis is listed in Table 1. These include single sites for androgen and estrogen response elements, CREB, AP-1, NF- $\mathrm{B}$, and several GATA-1 sites. Of particular interest to this study are three putative glucocorticoid response elements (GREs), one of which was identified by all three softwares $(-1675$ bp if TSS is + 1) and shares $13 / 15$ similarity with the GRE consensus sequence 5'-AGAACAnnnTGTTCT-3' (Malkoski \& Dorin 1999).

\section{Stressor exposure}

Zebrafish exposed to a 1-min air exposure stressor had $7 \cdot 0$-fold higher cortisol levels at $20 \mathrm{~min}$ recovery compared to unstressed control fish $(17 \cdot 5 \pm 5 \cdot 4$ vs $2 \cdot 5 \pm 0 \cdot 7 \mathrm{ng} / \mathrm{g}$ body weight; $P<0 \cdot 05, n=5-6)$. After longer recovery periods of 4 and 24 h, whole-body cortisol was not significantly different from control fish, nor was it significantly different from the $20 \mathrm{~min}$ recovery group (Fig. 4A). The mRNA abundance of $h s d 11 b 2$ in the brain following an air exposure stressor significantly decreased $(n=5 ; P<0 \cdot 06)$ at 20 min recovery relative to controls (Fig. 4B). Enzymatic conversion of ${ }^{3} \mathrm{H}$-cortisol to ${ }^{3} \mathrm{H}$-cortisone was significantly increased at 1 , 4 , and 24 h poststressor exposure relative to controls (Fig. 4C; $P<0 \cdot 05, n=4)$.

Incubation of ovary homogenate with ${ }^{3} \mathrm{H}$-cortisol for $4 \mathrm{~h}$ resulted in a total conversion of $85 \%$ to ${ }^{3} \mathrm{H}$-cortisone. Total cortisol conversion was markedly decreased $7 \cdot 5$-fold to $11 \%$ in the presence of the Hsd11b2 inhibitor, $18 \beta-G A$ (data not shown). This specificity was confirmed in brain homogenate, where incubations with inhibitor resulted in $83 \%$ reduction in cortisol conversion relative to controls

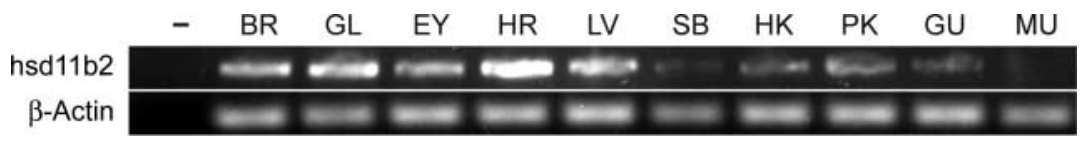

Figure 1 Expression of $h s d 11 b 2$ in various tissues of adult zebrafish, as determined by RT-PCR. As a positive control, $\beta$-actin was also amplified, and a no-template reaction was included as a negative control (-). Tissues are indicated at the top of each lane: BR, brain; GL, gill; EY, eye; HR, heart; LV, liver; SB, swim bladder; HK, head kidney; PK, posterior kidney; GU, gut; MU, muscle. 

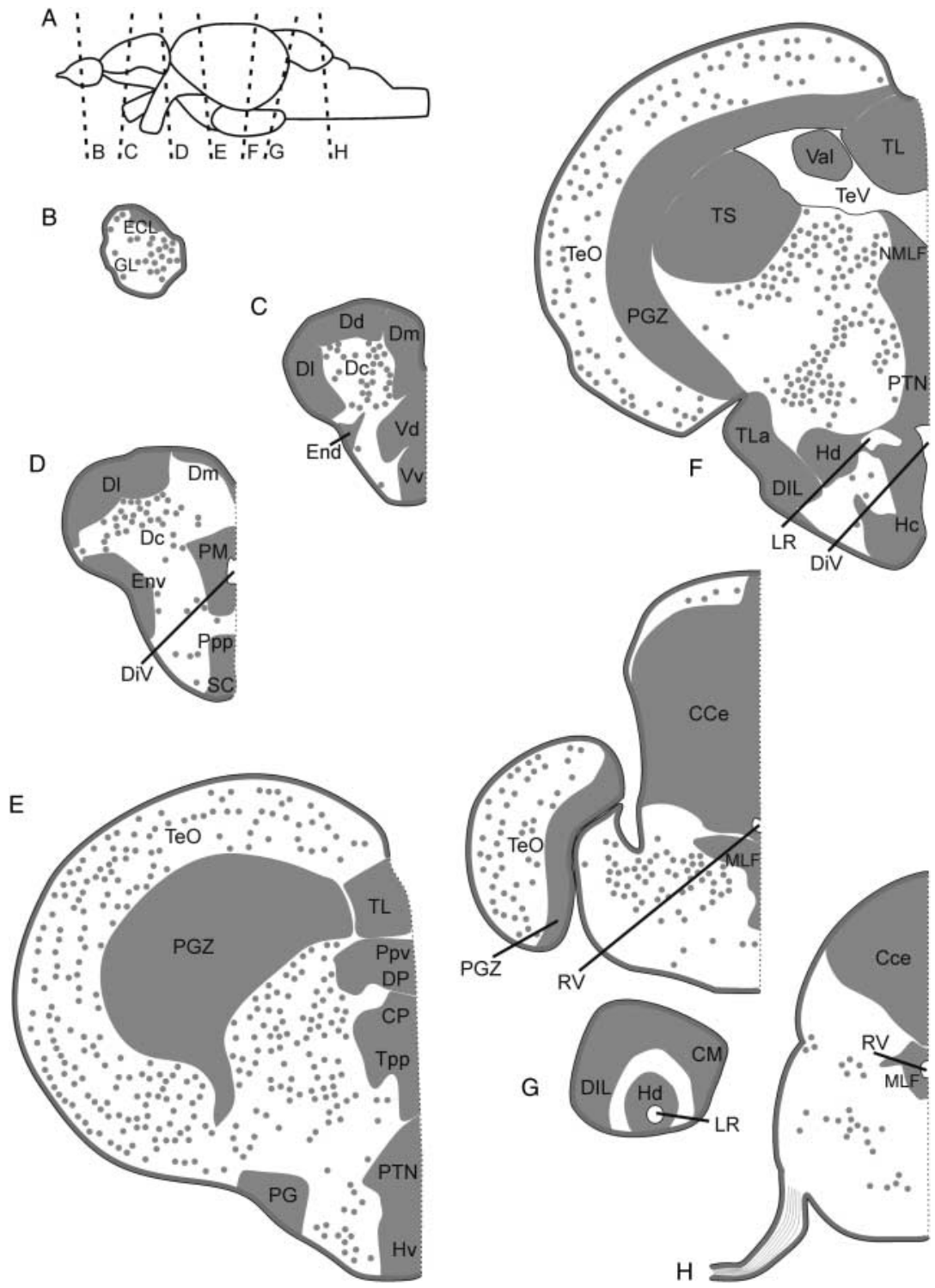

Figure 2 Composite of $h s d 11 b 2$ distribution in select regions of the zebrafish brain. Line drawings were traced from original photomicrographs of transverse sections following in situ hybridization. Gray circles indicate hybridization signal where a punctate expression pattern was observed; gray-shaded areas indicate regions of diffuse staining or extensive/overlapping punctate staining. (A) Schematic of a whole zebrafish brain, sagittal view, showing the approximate plane of section for B, C, D, E, F, G and H. For reference, the corresponding levels in the zebrafish brain atlas (Wullimann et al. 1996) are (B) 23, (C) 85, (D) 107-114, (E) 136-149, (F) 162-168, (G) 185-223, and (H) 239. For abbreviations, see list. Scale bar $=200 \mu \mathrm{m}$ for B, C, D, E, F, G and H. CCe, corpus cerebelli; CM, corpus mamillare; CP, central posterior thalamic nucleus; D, dorsal telencephalic area; Dc, central zone of D; Dd, dorsal zone of D; DIL, diffuse nucleus of the inferior lobe; DiV, diencephalic ventricle; DI, lateral zone of D; Dm, medial zone of D; DP, dorsal posterior thalamic nucleus; DTN, dorsal tegmental nucleus; ECL, external cellular layer of olfactory bulb; End, entopeduncular nucleus, dorsal part; ENv, entopeduncular nucleus, ventral part; GL, glomerular layer of olfactory bulb; Hc, caudal zone of periventricular hypothalamus; Hd, dorsal zone of periventricular hypothalamus; Hv, ventral zone of periventricular hypothalamus; LR, lateral recess of diencephalic ventricle; MLF, medial longitudinal fascicle; NMLF, nucleus of MLF; PG, preglomerular nucleus; PGZ, periventricular gray zone of the optic tectum;

PM, magnocellular preoptic nucleus; PPp, parvocellular preoptic nucleus, posterior part; PPv, periventricular pretectal nucleus, ventral part; $\mathrm{PTN}$, posterior tuberal nucleus; RV, rhombencephalic ventricle; $\mathrm{TeO}$, tectum opticum; TeV, tectal ventricle; TL, torus longitudinalis; TPp, periventricular nucleus of posterior tuberculum; TS, torus semicircularis; $\mathrm{V}$, ventral telencephalic area; Val, lateral division of valvula cerebelli; $\mathrm{Vd}$, dorsal nucleus of $\mathrm{V}$; $\mathrm{VI}$, lateral nucleus of $\mathrm{V} ; \mathrm{V} \mathrm{v}$, ventral nucleus of $\mathrm{V}$. 

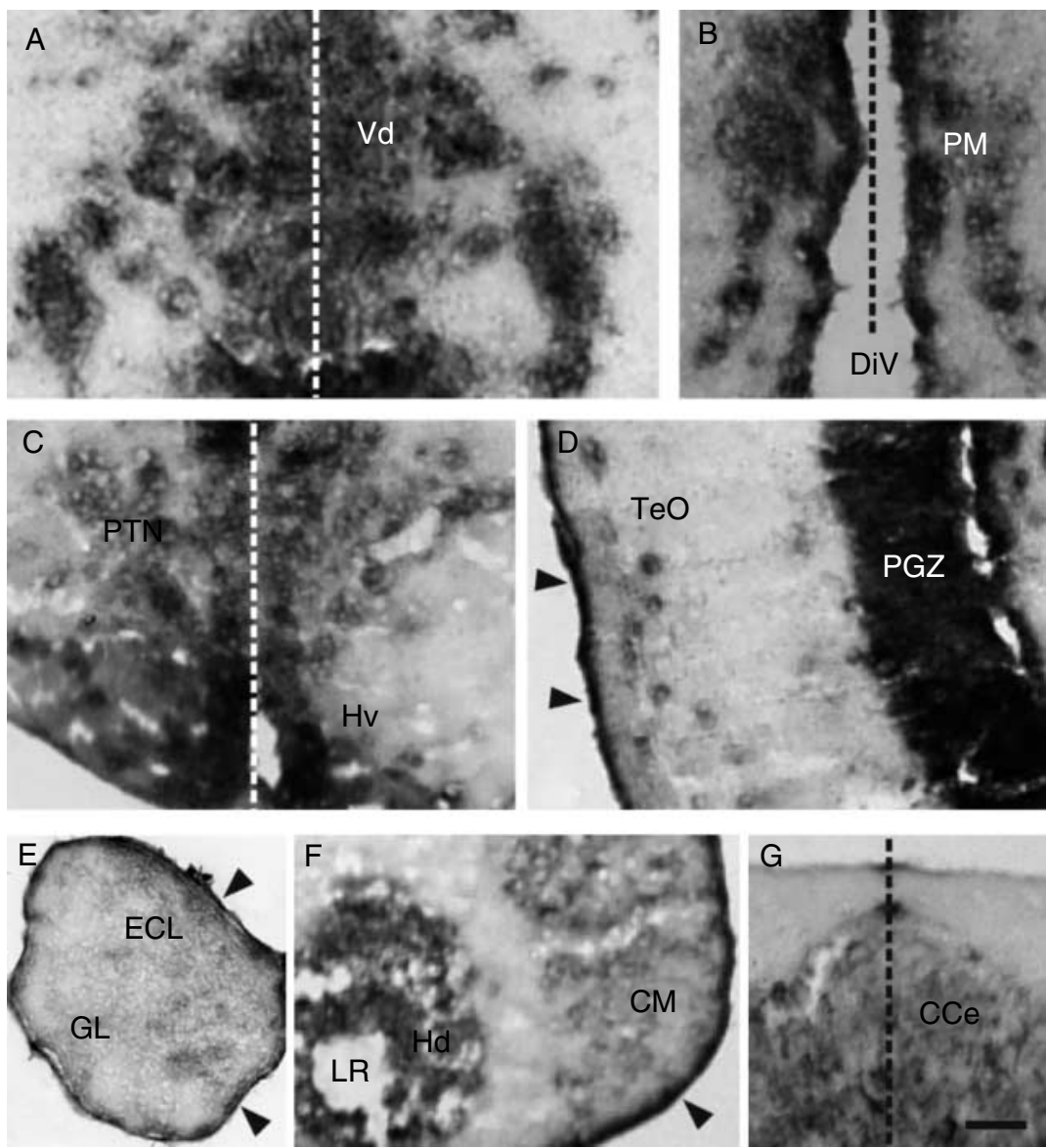

Figure 3 Brightfield photomicrographs of $h s d 11 \mathrm{~b} 2$ expression in transverse sections of adult zebrafish brains, as revealed by in situ hybridization. (A) Dorsal nucleus of the ventral telencephalic area $(\mathrm{Vd})$; (B) magnocellular preoptic nucleus (PM) on either side of the diencephalic ventricle (DiV); (C) posterior tuberal nucleus (PTN) and ventral zone of the periventricular hypothalamus $(\mathrm{Hv})$; (D) tectum opticum $(\mathrm{TeO})$ and periventricular gray zone (PGZ) of the optic tectum; (E) olfactory bulb; (F) corpus mamillare (CM) and diffuse nucleus (DIL) of the inferior lobe of the hypothalamus, LR, lateral recessus; $(\mathrm{G})$ corpus cerebelli (CCe). Vertical dashed lines indicate midlines of the sections, where appropriate. Arrowheads indicate positive hybridization signal along the periphery of the brain sections. Scale bar $=50 \mu \mathrm{m}$.

(Fig. 4D). Similarly, in vivo water-borne exposure to $18 \beta-G A$ decreased cortisol conversion by $47 \%$ in the brain of experimental fish relative to controls.

Analysis of whole-body cortisol in fish exposed to $18 \beta-$ GA showed an overall significant treatment effect, with $18 \beta$-GA exposed fish having, on average, $3 \cdot 8$-fold higher whole-body cortisol levels than solvent-exposed control fish (Fig. 5A; $P<0 \cdot 05)$. This difference was most pronounced in unstressed fish $(9 \cdot 3 \pm 3.9$ vs $1.3 \pm 0.52 \mathrm{ng} / \mathrm{g}$ tissue; $P<0 \cdot 05, n=6)$. Similarly, orf expression levels in the POA of $18 \beta-G A$-exposed fish were, on average, 1.2-fold higher than controls (Fig. 5B; $P<0 \cdot 05)$, with the difference being most pronounced in unstressed fish $(P<0 \cdot 05, n=5-6)$. While there were no differences in POA $g r$ expression between treatment groups (Fig. 5C), there was a significant interaction between treatment and time for $m r$ (Fig. 5D; $P<0 \cdot 01, n=6$ ).
In particular, control fish at $24 \mathrm{~h}$ poststress had 1.7-fold higher $m r$ expression in the POA than control fish at $0 \mathrm{~h}$ (Fig. 5D; $P<0 \cdot 001, n=6$ ), and 18ß-GA-exposed fish at $0 \mathrm{~h}$ had $1 \cdot 7$-fold higher expression compared with the control fish (Fig. 5D; $P<0 \cdot 001, n=6$ ).

\section{Discussion}

This study presents the first thorough description of Hsd11b2 expression and activity in the CNS of a nonmammalian vertebrate. Notably, hsd $11 b 2$ transcripts are widespread in the adult zebrafish brain, reminiscent of the distribution pattern seen in the fetal brain of mammals, but in sharp contrast to adults (Wyrwoll et al. 2011). We also present novel evidence that central Hsd11b2 may be playing a critical role in the 
Table 1 Selected putative transcription factor response elements for Hsd11b2 identified in the 2434 bp upstream of the TSS $(+1)$. The position of each response element was confirmed by at least two of the three softwares used in the promoter analysis

\section{Transcription factor response element}

Androgen response element

AP-1

CREB

Estrogen response element

GATA-1

Glucocorticoid response element $\mathrm{NF}-\kappa \mathrm{B}$

Location $(b p)$

$$
\begin{aligned}
& -459 \\
& -774 \\
& -1283 \\
& -1699 \\
& -119,-1100,-1848, \\
& \quad-1939 \\
& -942,-1675,-2398 \\
& -359
\end{aligned}
$$

negative feedback regulation of cortisol during stress in zebrafish.

\section{Distribution and functional implications}

The distribution of $h s d 11 b 2$ in the CNS described in this study (Figs 2 and 3 ) is far more extensive than what has been previously reported in adult mammals (Robson et al. 1998,
Geerling et al. 2006). Also in adult birds, although $h s d 11 b 2$ mRNA was detected in several brain regions, enzymatic activity was low in the zebra finch (Taeniopygia guttata; Katz et al. 2010) and undetectable in chicken brain (Gallus gallus; Klusonová et al. 2008). By contrast, we observed substantial enzymatic activity of Hsd11b2 in adult zebrafish brain homogenates, the first such description in teleosts. This suggests that the developmental loss of brain Hsd11b2 observed in higher vertebrates may not occur in fish, while the functional relevance of this observation from an evolutionary standpoint awaits further study.

Interestingly, the pattern of $h s d 11 b 2$ expression in the brain reported in this study parallels the distribution of key steroidogenic enzymes in the adult zebrafish brain (Diotel et al. 2010, 2011), including prominent expression in the telencephalon (Fig. 3A) and peripheral edges of the brain (Fig. 3D, E and F). These authors propose that neurosteroidogenesis plays a key role in maintaining the persistent neural proliferation observed in adult fish. In support of this hypothesis, the distribution patterns of these enzymes coincide with the described neurogenic regions of the zebrafish brain (Zupanc et al. 2005, Grandel et al. 2006). In fetal mammals, Hsd11b2 prevents untimely neural

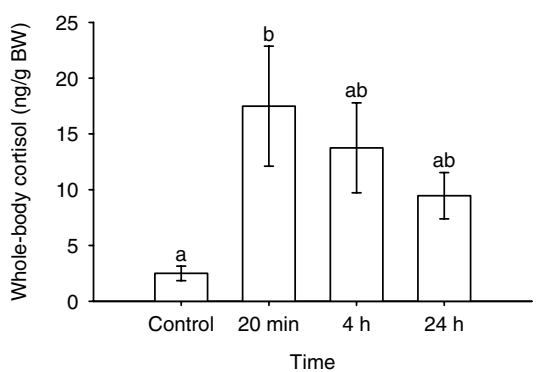

C

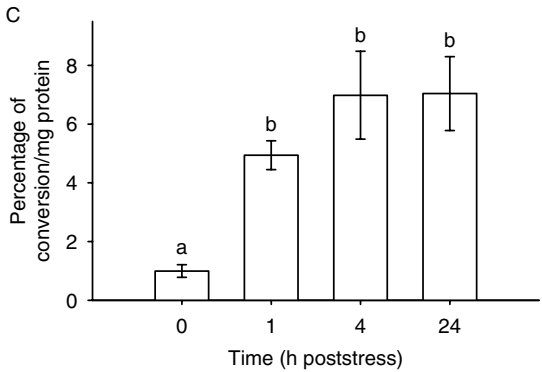

B
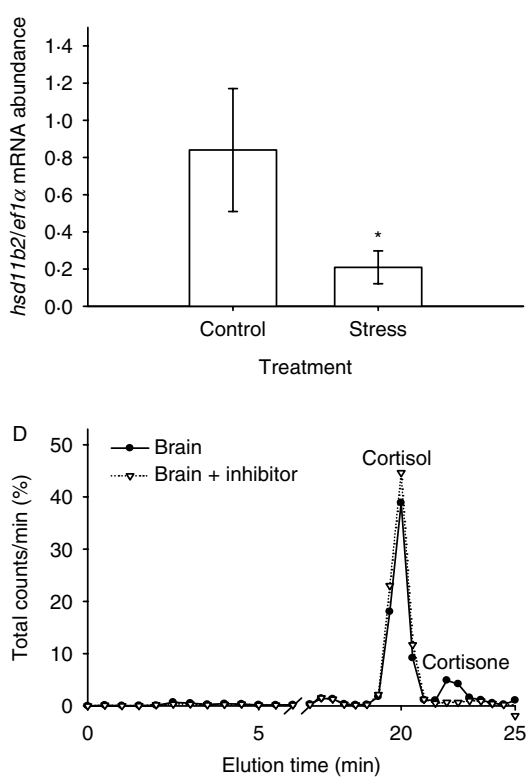

Figure 4 Effect of a 1-min air exposure stressor on (A) whole-body cortisol, quantified by ELISA; (B) hsd11b2 gene expression in the brain after 20-min recovery, as determined by qPCR and normalized to the expression of ef $1 \alpha$ and (C) Hsd11b2 enzymatic activity (percentage of conversion/mg protein) in the brain after 1-, 4-, and 24-h recovery, as determined by quantifying ${ }^{3} \mathrm{H}$-cortisol conversion to ${ }^{3} \mathrm{H}$-cortisone using $\mathrm{HPLC}$.

(D) Representative graph of HPLC results showing the relative amounts of ${ }^{3} \mathrm{H}$-cortisol and ${ }^{3} \mathrm{H}$-cortisone in brain homogenates alone (black circles) or in combination with the Hsd11b2 inhibitor, $18 \beta$-glycyrrhetinic acid (white triangles), expressed as percentage of total c.p.m. Values are presented as mean \pm S.E.M. $(n=4-6)$. Differences in whole-body cortisol and enzymatic activity were determined using a one-way ANOVA and letters indicate significance $(P<0 \cdot 05)$. A change in gene expression was determined with a Student's $t$-test and significance is denoted with an asterisk $(P<0 \cdot 06)$. 


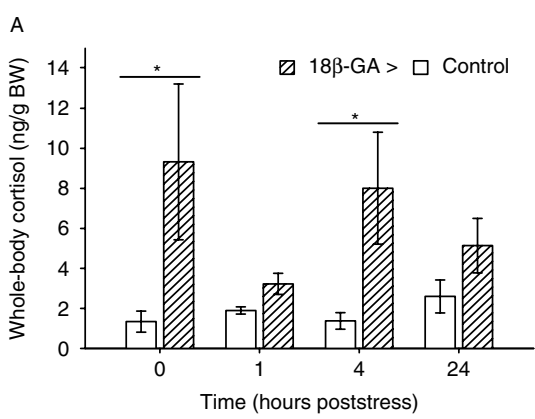

B
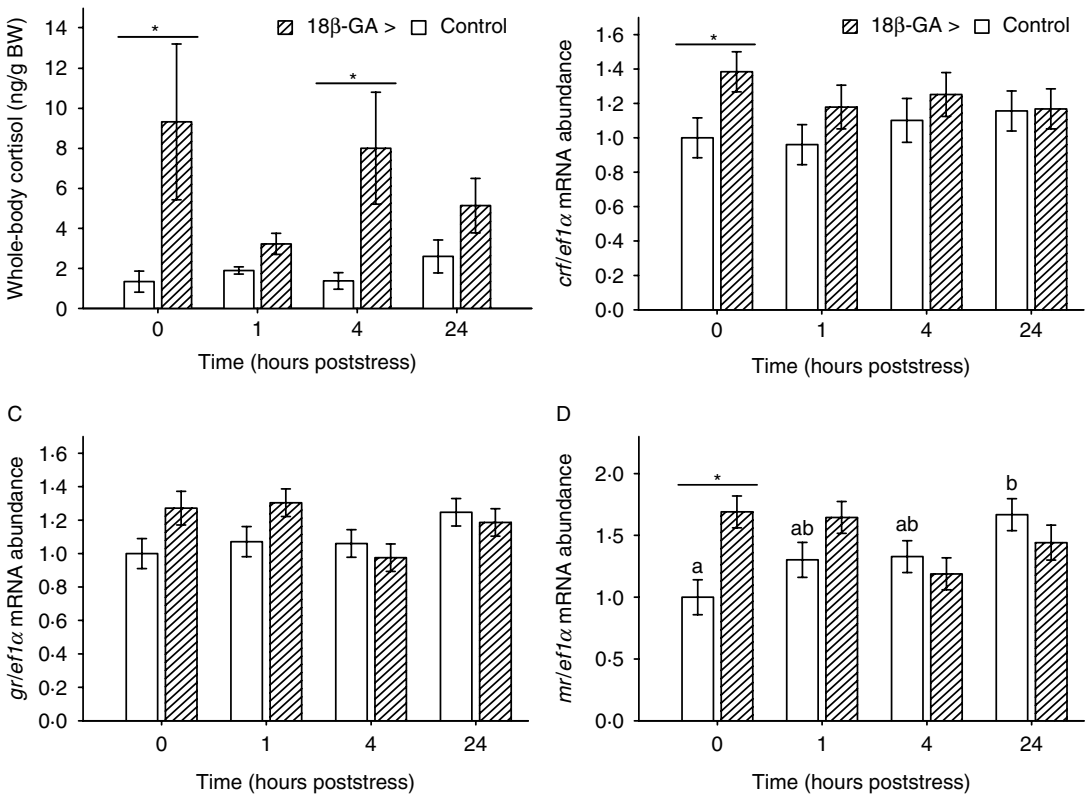

$\mathrm{D}$

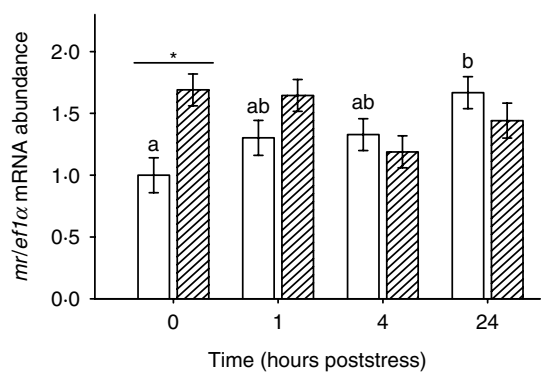

Figure 5 Effects of Hsd11b2 inhibition using water-borne exposure to 18ß-GA on (A) wholebody cortisol content, and preoptic area gene expression of (B) $c r f,(C) g r$, and (D) $m r$. Fish were exposed to vehicle control $(0 \cdot 1 \%$ ethanol; white bars) or $18 \beta-\mathrm{GA}(1 \mathrm{mg} / \mathrm{l}$; hatched bars $)$ for $48 \mathrm{~h}$ and then sampled immediately $(0 \mathrm{~h})$ or subjected to air exposure for $1 \mathrm{~min}$ and allowed to recover for 1, 4, or $24 \mathrm{~h}$. Whole-body cortisol content was quantified by ELISA and is expressed as nanogram per gram body weight, and relative abundance of target gene mRNA normalized to the expression of ef $1 \alpha$ was obtained using qPCR. Values are presented as mean \pm s.E.M. $(n=5-6)$. Changes in cortisol and transcript levels were determined using a two-way ANOVA $(P<0 \cdot 05)$; significant differences between treatments within a given time point are indicated by an asterisk, and different letters denote temporal differences within a given treatment; overall differences between treatments across all time points are noted as insets.

differentiation by glucocorticoids and is required for normal brain development (Diaz et al. 1998, Robson et al. 1998, Holmes et al. 2006). Therefore, the extensive distribution and activity of Hsd11b2 in the adult zebrafish brain leads us to propose that persistent neurogenesis in adult zebrafish is achieved, in part, by Hsd11b2 mitigating excess glucocorticoid-mediated impact on differentiation, but this remains to be tested.

Teleost peripheral tissues broadly express hsd11b2 (Jiang et al. (2003), Kusakabe et al. (2003) and Rasheeda et al. (2010), this study). This contrasts peripheral expression in mammals and birds, where Hsd11b2 is primarily found in aldosterone target tissues such as the kidney, colon, and heart (Klusonová et al. 2008, Katz et al. 2010, Wyrwoll et al. 2011). We hypothesize that the absence of a corticosteroid binding globulin (CBG) in fish plasma may explain this phylogenetic difference. Up to $95 \%$ of circulating glucocorticoids is bound to $\mathrm{CBG}$ and is not available for biological activity in higher vertebrates, whereas there is no supporting evidence of such regulation in fish (Mommsen et al. 1999). In addition to reducing GR protein content in peripheral tissues following stress (Alderman et al. 2012, Ings et al. 2012), peripheral expression of Hsd11b2 would allow cells an alternative mechanism for regulating local cortisol levels in the absence of a CBG. This notion is supported by our observation of elevated whole-body cortisol in unstressed zebrafish exposed to $18 \beta-$ GA and warrants further investigation.

\section{Hsd11b2 and HPI axis regulation}

The consistent increase in Hsd11b2 brain activity following acute air exposure suggests a role for this enzyme in regulating cortisol signaling poststress in the zebrafish brain (Fig. 4C). This increased enzyme activity may involve enhanced mRNA turnover and translation given the reduction in hsd11b2 transcript levels at $20 \mathrm{~min}$ poststress (Fig. 4B). We hypothesize that stressor-induced elevation in cortisol levels regulate central $h s d 11 b 2$ expression in zebrafish. In support, our promoter analysis identified three putative GREs by which GR signaling may regulate $h s d 11 b 2$ gene expression (Table 1). Also, ligand-bound GR has been shown to interact with other transcription factors, including AP-1 (Malkoski \& Dorin 1999) and NF-KB (Liden et al. 1997), whose putative response element sites were identified on zebrafish $h s d 11 b 2$ promoter, to affect gene transcription. In addition, recent studies showed an upregulation of $h s d 11 b 2$ in the head kidney after stressor exposure, which also resulted in elevated cortisol levels, in zebrafish, and common carp (Cyprinus carpio), 
suggesting transcriptional regulation (Nematollahi et al. 2009, Fuzzen et al. 2010). However, more studies are needed to tease out the direction, timing, and tissue specificity of $h s d 11 b 2$ regulation by cortisol.

The stimulatory effect of stress on Hsd11b2 activity observed in Experiment 1 combined with the putative regulation of $h s d 11 b 2$ gene expression by cortisol fits well with the negative feedback model of HPI axis regulation and is further supported by results from Experiment 2. Adult zebrafish exposed to the Hsd11b2 enzyme inhibitor, $18 \beta$-GA, had higher basal and poststress cortisol content compared with control fish (Fig. 5A). While this observation may relate in part to the contribution of peripheral tissues in cortisol deactivation, changes in POA gene expression support involvement of central Hsd11b2 in basal cortisol regulation, and $h s d 11 b 2$ is expressed in the POA (Fig. 3B). POA $c r f$ mRNA abundance was significantly higher in 18ß-GA-exposed fish (Fig. 5B), consistent with hyperactivation of the HPI axis and implicating Hsd11b2 in the transcriptional regulation of CRF by cortisol. Chronic elevations in cortisol are typically associated with decreased POA orf mRNA abundance due to the hypothalamic negative feedback loop (Bernier et al. 2004). However, in unstressed rainbow trout, RU486 (GR antagonist) decreased POA crf mRNA abundance (Alderman et al. 2012), suggesting that cortisol signaling at basal levels is required to sustain the $c r f$ mRNA pool. This agrees with our finding that in nonstressed zebrafish, local increases in brain cortisol levels caused by Hsd11b2 inhibition increased crfmRNA abundance (Fig. 5B).

In mammals, low levels of glucocorticoids acting at MR in the limbic system modulate basal (diurnal) HPA axis activity, whereas high levels of glucocorticoids suppress stressorinduced activation of the HPA axis in part through GR-mediated suppression of $c r f$ transcript abundance in the POA (Yao \& Denver 2007). In fish, the relative contribution of GR and MR to HPI axis regulation is less clear. We recently showed that GR is required for normal and stressinduced HPI axis activity in rainbow trout (Alderman et al. 2012), yet exposure to stressors does not alter GR protein or transcript abundances in the teleost brain (Alderman et al. (2012) and Ings et al. (2012), this study). We propose, based on our observation, that Hsd11b2 activity in the brain is increased by stress and that GR signaling is constrained by local ligand availability (i.e. cortisol inactivation) rather than receptor abundance in fish. Whether activation of Hsd11b2 activity is mediated by GR or MR is not clear, but our observation of higher $m r$ mRNA abundance in the POA following stress and in unstressed fish exposed to 18ß-GA (Fig. 5D) supports involvement of $\mathrm{MR}$ in this role.

\section{Conclusions}

Our results underscore the importance of central Hsd11b2 regulation in HPI axis functioning, including the hypothalamic negative feedback loop for cortisol in response to stress in fish. The mechanism leading to the control of cortisol dynamics by Hsd11b2 is unclear, but our results suggest that localized lowering of brain cortisol levels by this enzyme may be involved in the suppression of $r f$ transcript abundance in the POA, in turn leading to reduced HPI axis activity poststress in fish. The widespread distribution of $h s d 11 b 2$ in adult zebrafish brain, and the increased activity of this enzyme in response to stress, further supports a key role for Hsd11b2 in reducing central cortisol levels after stress in adult zebrafish brain. We hypothesize a role for MR, based on changes in transcript abundance, in the negative feedback regulation of cortisol poststress. As cortisol stimulation is thought to play a very important role in neural differentiation, we hypothesize that deactivation of excess glucocorticoid by Hsd11b2 may be of functional relevance in neural proliferation and brain function in teleosts.

\section{Declaration of interest}

The authors declare that there is no conflict of interest that could be perceived as prejudicing the impartiality of the research reported.

\section{Funding}

This study was funded by the Natural Sciences and Engineering Research Council of Canada Discovery Grant (217481-2008) and the Discovery Accelerator Supplement (364396-2008) to M M V.

\section{Acknowledgements}

The authors thank Dr V Choh (University of Waterloo) for use of her cryostat, Drs J Leatherland and H Christie (University of Guelph) for HPLC analysis, C Best, N Melnyk-Lamont, and D Nesan (University of Waterloo) for help with sampling.

\section{References}

Alderman SL \& Bernier NJ 2007 Localization of corticotropin-releasing factor, urotensin I, and CRF-binding protein gene expression in the brain of the zebrafish, Danio rerio. Journal of Comparative Neurology 502 783-793. (doi:10.1002/cne.21332)

Alderman SL, McGuire A, Bernier NJ \& Vijayan MM 2012 Central and peripheral glucocorticoid receptors are involved in the plasma cortisol response to an acute stressor in rainbow trout. General and Comparative Endocrinology 176 79-85. (doi:10.1016/j.ygcen.2011.12.031)

Alsop D \& Vijayan MM 2008 Development of the corticosteroid stress axis and receptor expression in zebrafish. American Journal of Physiology. Regulatory, Integrative and Comparative Physiology 294 R711-R719. (doi:10.1152/ajpregu.00671.2007)

Aluru N, Renaud R, Leatherland JF \& Vijayan MM 2005 Ah receptormediated impairment of interrenal steroidogenesis involves StAR protein and $\mathrm{P} 450 \mathrm{scc}$ gene attenuation in rainbow trout. Toxicological Sciences 84 260-269. (doi:10.1093/toxsci/kfi077)

Bernier NJ, Bedard N \& Peter RE 2004 Effects of cortisol on food intake, growth, and forebrain neuropeptide $\mathrm{Y}$ and corticotropin-releasing factor gene expression in goldfish. General and Comparative Endocrinology 135 230-240. (doi:10.1016/j.ygcen.2003.09.016) 
Colombo L, Dalla Valle L, Fiore C, Armanini D \& Belvedere P 2006 Aldosterone and the conquest of land. Journal of Endocrinological Investigation 29 373-379.

Diaz R, Brown RW \& Seckl JR 1998 Distinct ontogeny of glucocorticoid and mineralocorticoid receptor and $11 \beta$-hydroxysteroid dehydrogenase types I and II mRNAs in the fetal rat brain suggest a complex control of glucocorticoid actions. Journal of Neuroscience 18 2570-2580.

Diotel N, Le Page Y, Mouriec K, Tong SK, Pellegrini E, Vaillant C, Anglade I, Brion F, Pakdel F, Chung BC et al. 2010 Aromatase in the brain of teleost fish: expression, regulation and putative functions. Frontiers in Neuroendocrinology 31 172-192. (doi:10.1016/j.yfrne.2010.01.003)

Diotel N, Do Rego J-L, Anglade I, Vaillant C, Pellegrini E, Gueguen M-M, Mironov S, Vaudry H \& Kah O 2011 Activity and expression of steroidogenic enzymes in the brain of adult zebrafish. European Journal of Neuroscience 34 45-56. (doi:10.1111/j.1460-9568.2011.07731.x)

Fryer JN \& Peter RE 1977 Hypothalamic control of ACTH secretion in goldfish. III. Hypothalamic cortisol implant studies. General and Comparative Endocrinology 33 215-225. (doi:10.1016/0016-6480(77)90246-5)

Fuzzen ML, Van Der Kraak G \& Bernier NJ 2010 Stirring up new ideas about the regulation of the hypothalamic-pituitary-interrenal axis in zebrafish (Danio rerio). Zebrafish 7 349-358. (doi:10.1089/zeb.2010.0662)

Geerling JC, Kawata M \& Loewy AD 2006 Aldosterone-sensitive neurons in the rat central nervous system. Journal of Comparative Neurology 494 515-527. (doi:10.1002/cne.20808)

Gonçalves AF, Páscoa I, Neves JV, Coimbra J, Vijayan MM, Rodrigues P \& Wilson JM 2012 The inhibitory effect of environmental ammonia on Danio rerio LPS induced acute phase response. Developmental and Comparative Immunology 36 279-288. (doi:10.1016/j.dci.2011.04.008)

Grandel H, Kaslin J, Ganz J, Wenzel I \& Brand M 2006 Neural stem cells and neurogenesis in the adult zebrafish brain: origin, proliferation dynamics, migration and cell fate. Developmental Biology 295 263-277. (doi:10.1016/ j.ydbio.2006.03.040)

Holmes MC, Abrahamsen CT, French KL, Paterson JM, Mullins JJ \& Seckl JR 2006 The mother or the fetus? 11 $\beta$-Hydroxysteroid dehydrogenase type 2 null mice provide evidence for direct fetal programming of behavior by endogenous glucocorticoids. Journal of Neuroscience $\mathbf{2 6}$ 3840-3844. (doi:10.1523/JNEUROSCI.4464-05.2006)

Ings JS, Oakes KD, Vijayan MM \& Servos MR 2012 Temporal changes in stress and tissue-specific metabolic responses to municipal wastewater effluent exposure in rainbow trout. Comparative Biochemistry and Physiology. Part C: Toxicology \& Pharmacology 156 67-74. (doi:10.1016/j.cbpc.2012.04.002)

Jiang JQ, Wang DS, Senthilkumaran B, Kobayashi T, Kobayashi HK, Yamaguchi A, Ge W, Young G \& Nagahama Y 2003 Isolation, characterization and expression of $11 \beta$-hydroxysteroid dehydrogenase type 2 cDNAs from the testes of Japanese eel (Anguilla japonica) and Nile tilapia (Oreochromis niloticus). Journal of Molecular Endocrinology 31 305-315. (doi:10.1677/jme.0.0310305)

Katz A, Oyama RK, Feng N, Chen X \& Schlinger BA 2010

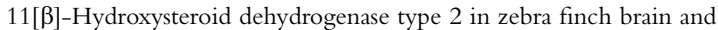
peripheral tissues. General and Comparative Endocrinology 166 600-605. (doi:10.1016/j.ygcen.2010.01.016)

Klusonová P, Kucka M, Miksík I, Bryndová J \& Pácha J 2008 Chicken $11[\beta]$-hydroxysteroid dehydrogenase type 2 : partial cloning and tissue distribution. Steroids 73 348-355. (doi:10.1016/j.steroids.2007.11.006)

Krozowski ZS 1999 The $11 \beta$-hydroxysteroid dehydrogenases: functions and physiological effects. Molecular and Cellular Endocrinology 151 121-127. (doi:10.1016/S0303-7207(98)00256-1)

Kusakabe M, Nakamura I \& Young G 2003 11ß-Hydroxysteroid dehydrogenase complementary deoxyribonucleic acid in rainbow trout: cloning, sites of expression, and seasonal changes in gonads. Endocrinology 144 2534-2545. (doi:10.1210/en.2002-220446)

Liden J, Delaunay F, Rafter I, Gustafsson J \& Okret S 1997 A new function for the C-terminal zinc finger of the glucocorticoid receptor. Repression of RelA transactivation. Journal of Biological Chemistry 272 21467-21472. (doi:10.1074/jbc.272.34.21467)
Lowartz S, Petkam R, Renaud R, Beamish FW, Kime DE, Raeside J \& Leatherland JF 2003 Blood steroid profile and in vitro steroidogenesis by ovarian follicles and testis fragments of adult sea lamprey, Petromyzon marinus. Comparative Biochemistry and Physiology. Part A, Molecular \& Integrative Physiology 134 365-376. (doi:10.1016/S1095-6433(02)00285-4)

Malkoski SP \& Dorin RI 1999 Composite glucocorticoid regulation at a functionally defined negative glucocorticoid response element of the human corticotropin-releasing hormone gene. Molecular Endocrinology 13 1629-1644. (doi:10.1210/me.13.10.1629)

Mommsen TP, Vijayan MM \& Moon TW 1999 Cortisol in teleosts: dynamics, mechanisms of action, and metabolic regulation. Reviews in Fish Biology and Fisheries 9 211-268. (doi:10.1023/A:1008924418720)

Nematollahi MA, van Pelt-Heerschap H \& Komen J 2009 Transcript levels of five enzymes involved in cortisol synthesis and regulation during the stress response in common carp: relationship with cortisol. General and Comparative Endocrinology 164 85-90. (doi:10.1016/j.ygcen.2009.05.006)

Rasheeda MK, Kagawa H, Kirubagaran R, Dutta-Gupta A \& Senthilkumaran B 2010 Cloning expression and enzyme activity analysis of testicular $11 \beta$-hydroxysteroid dehydrogenase during seasonal cycle and after hCG induction in air-breathing catfish, Clarias gariepinus. Journal of Steroid Biochemistry and Molecular Biology 120 1-10. (doi:10.1016/j.jsbmb. 2010.02.014)

Robson AC, Leckie CM, Seckl JR \& Holmes MC 1998 11ß-Hydroxysteroid dehydrogenase type 2 in the postnatal and adult rat brain. Brain Research. Molecular Brain Research 61 1-10. (doi:10.1016/S0169-328X(98)00161-2)

Stolte EH, de Mazon AF, Leon-Koosterziel KM, Jesiak M, Bury NR, Sturm A, Savelkoul HF, van Kemenade BM \& Flik G 2008 Corticosteroid receptors involved in stress regulation in common carp, Cyprinus carpio. Journal of Endocrinology 198 403-417. (doi:10.1677/JOE-08-0100)

Sturm A, Bury N, Dengreville L, Fagart J, Flouriot G, Rafestin-Oblin ME \& Prunet P 2005 11-Deoxycorticosterone is a potent agonist of the rainbow trout (Oncorhynchus mykiss) mineralocorticoid receptor. Endocrinology 146 47-55. (doi:10.1210/en.2004-0128)

Thisse C \& Thisse B 2008 High-resolution in situ hybridization to wholemount zebrafish embryos. Nature Protocols 3 59-69. (doi:10.1038/nprot. 2007.514)

Vicker N, Su X, Lawrence H, Cruttenden A, Purohit A, Reed MJ \& Potter BV 2004 A novel 18ß-glycyrrhetinic acid analogue as a potent and selective inhibitor of $11 \beta$-hydroxysteroid dehydrogenase 2. Bioorganic $\mathcal{E}$ Medicinal Chemistry 14 3263-3267. (doi:10.1016/j.bmcl.2004.03.107)

Wendelaar Bonga SE 1997 The stress response in fish. Physiological Reviews 77 591-625.

Wullimann MF, Rupp B \& Reichert H 1996. Neuroanatomy of the Zebrafish Brain: a Topological Atlas. Basel: Birkhauser.

Wyrwoll CS, Holmes MC \& Seckl JR 2011 11ß-Hydroxysteroid dehydrogenases and the brain: from zero to hero, a decade of progress. Frontiers in Neuroendocrinology 32 265-286. (doi:10.1016/j.yfrne.2010. 12.001)

Yao M \& Denver RJ 2007 Regulation of vertebrate corticotropin-releasing factor genes. General and Comparative Endocrinology 153 200-216. (doi:10.1016/j.ygcen.2007.01.046)

Zupanc GK, Hinsch K \& Gage FH 2005 Proliferation, migration, neuronal differentiation, and long-term survival of new cells in the adult zebrafish brain. Journal of Comparative Neurology 488 290-319. (doi:10.1002/cne. 20571)

\section{Received in final form 2 October 2012 Accepted 5 October 2012 Made available online as an Accepted Preprint 5 October 2012}

